\title{
Avaliação da qualidade de vida antes e depois de tratamento fisioterapêutico para incontinência urinária
}

\author{
Quality of life assessment before and after \\ a physical therapy intervention for urinary incontinence
}

Evaluación de la calidad de vida antes y después

de tratamiento fisioterapéutico para incontinencia

Mara Regina Knorst', Camila de Souza Royer', Daiane Marcelle da Silva Basso', Juliano dos Santos Russo', Roberta Giacobbo Guedes ${ }^{1}$, Thais de Lima Resende ${ }^{1}$

RESUMO I Esse estudo quase experimental analisou a influência de tratamento fisioterapêutico na qualidade de vida (QV) de mulheres com incontinência urinária (IU), bem como a sua eficácia na perda urinária. Participaram 55 mulheres (35 a 87 anos) com o diagnóstico médico de IU, as quais, antes da intervenção, se submeteram a uma anamnese e tiveram avaliadas a função da musculatura do assoalho pélvico (FMAP; teste bidigital) e a QV (King's Health Questionnaire $\mathrm{KHQ}$ ). Foram realizadas até 15 sessões (uma por semana) com eletroestimulação endovaginal e treino da musculatura do assoalho pélvico. Assim que as participantes relatavam não mais perder urina ou manifestavam o desejo de interromper o tratamento, o mesmo era terminado, independente do número de sessões. Após o tratamento, além de reaplicar o teste bidigital e o KHQ, foi também perguntado às participantes se consideravam-se continentes, satisfeitas com o tratamento ou não perceberam melhora. Predominaram mulheres com idades entre 51 e 60 anos que realizaram parto normal com episiotomia e apresentaram prolapso. A maioria apresentou IU mista, seguida da de esforço. Tossir e espirrar foram as situações em que mais comumente ocorreu perda urinária, a qual se deu mais em jatos. Houve melhora significativa em todos os domínios da QV, exceto na percepção geral da saúde. Após a intervenção, 90,9\% delas se declaram continentes ou satisfeitas. O tratamento fisioterapêutico resultou em melhora da QV e foi eficaz para contenção da perda urinária.

Descritores I incontinência urinária; qualidade de vida; saúde da mulher.
ABSTRACT I This quasi experimental study analyzed the influence of a physical therapy intervention on the quality of life (QoL) of women with urinary incontinence (UI) and its effectiveness for urinary loss. Took part in this study 55 women (35 to 87 years) with a clinical diagnosis of UI who underwent an anamnesis and had the function of their pelvic floor muscles (PFMF; bidigital test) and their QoL measured (King's Health Questionnaire - KHQ). Up to 15 weekly sessions were carried out with endovaginal electrical stimulation and perineal exercises. Once the participants reported not losing urine or expressed the desire to stop the treatment, it was terminated, regardless of the number of sessions undertaken. After treatment, the bidigital test and the $\mathrm{KHQ}$ were repeated and the participants were asked if they considered themselves to be continent, satisfied with the treatment or not improved. Most participants were aged between 51 and 60 years, underwent vaginal delivery with episiotomy and had prolapse. Most had mixed UI, followed by stress Ul. The situations when the urinary loss most commonly occurred were coughing and sneezing, which happened mostly in jets. There was a significant improvement in all of the KHQ domains, except the general health perception. After the intervention, 90.9\% of the women said that they were continent or satisfied with the treatment. The physical therapy intervention resulted in improved QoL and it was effective for containing the urinary loss.

Keywords I urinary incontinence; quality of life; women's health.

Estudo desenvolvido na Faculdade de Enfermagem, Nutrição e Fisioterapia da Pontifícia Universidade Católica do Rio Grande do Sul (PUCRS) - Porto Alegre (RS), Brasil.

'PUCRS - Porto Alegre (RS), Brasil. 
RESUMEN I Este estudio casi experimental analizó la influencia de tratamiento fisioterapéutico en la calidad de vida (CV) de mujeres con incontinencia urinaria (IU), así como su eficacia en la pérdida urinaria. Participaron 55 mujeres (35 a 87 años) con diagnóstico médico de IU, las cuales, antes de la intervención, fueron sometidas a una anamnesis y se les evaluó la función de la musculatura del piso pélvico (FMAP; test bidigital) y la CV (King's Health Questionnaire - KHQ). Fueron realizadas hasta 15 sesiones (una por semana) con electroestimulación endovaginal y entrenamiento de la musculatura del piso pélvico. Cuando las participantes relataban que no perdían más perder orina o manifestaban el deseo de interrumpir el tratamiento, el mismo era terminado, independientemente del número de sesiones. Después del tratamiento, además de reaplicar el test bidigital y el KHQ, se les preguntó a las participantes si se consideraban continentes, satisfechas con el tratamiento o no notaron mejoría. Predominaron mujeres con edades entre 51 y 60 años que realizaron parto normal con episiotomía y presentaron prolapso. La mayoría presentó IU mixta, seguida de la de esfuerzo. Toser y estornudar fueron las situaciones en que más comúnmente ocurría pérdida urinaria, la cual sucedió más en chorros. Hubo mejora significativa en todos los dominios de la CV, excepto en la percepción general de la salud. Después de la intervención, 90,9\% de ellas se declaran continentes o satisfechas. El tratamiento fisioterapéutico resultó en mejora de la CV y fue eficaz para contención de la pérdida urinaria.

Palabras clave I incontinencia urinaria; calidad de vida; salud de la mujer.

\section{INTRODUÇÃO}

A incontinência urinária (IU) é um problema de saúde que afeta milhões de pessoas em todo o mundo ${ }^{1}$ em seus aspectos físicos, psicológicos, sociais, sexuais e ocupacionais ${ }^{2}$. Considerada um problema de saúde pública ${ }^{1}$, a sua prevalência média em mulheres é de 27,6 e em homens de $10,5 \%{ }^{3}$, sendo maior no gênero feminino devido às variações anatômicas. Apesar do crescimento de sua prevalência com o aumento da idade, esta condição não deve ser tratada como uma consequência natural ou mesmo exclusiva do envelhecimento ${ }^{4}$.

A IU foi definida como perda involuntária de urina, que é objetivamente demonstrável, sendo um problema social ou higiênico ${ }^{5}$. Entretanto, além de variação individual considerável no que cada pessoa classifica como "problema", existem muitos indivíduos com IU que ficam constrangidos em consultar seus médicos sobre o assunto, ou que consideram a sua condição inevitável ${ }^{5}$. Ela exerce múltiplos efeitos sobre atividades diárias, interações sociais e percepção própria de saúde. Os maiores problemas relacionam-se ao bem-estar social e mental, afetando de modo significativo a qualidade de vida (QV), com consequências psicológicas, físicas, profissionais, sexuais e sociais ${ }^{6}$.

Por sua vez, a QV está ligada à percepção do indivíduo sobre a sua vida familiar, amorosa, social, ambiental e suas condições de saúde ${ }^{7,8}$. O grau de impacto da IU na $Q V$ varia de acordo com o seu tipo e a sua gravidade ou com a percepção individual do problema ${ }^{8}$. Em relação às mulheres, elas sentem-se desconfortáveis e insatisfeitas com os diferentes impactos causados pela IU, como dificuldade em frequentar lugares públicos, dormir fora de casa e visitar amigos, levando-as a desistir de um convívio social ou até de uma vida sexual estável ${ }^{4,9}$.
Dado o impacto da IU na QV, a sua mensuração é um dos parâmetros úteis para avaliar o resultado do tratamento escolhido. As ferramentas para verificar a QV geralmente incluem aspectos gerais sobre a saúde e específicos sobre os efeitos que determinada doença ou disfunção causa sobre o estilo de vida de um indivíduo. Este segundo aspecto seria mais sensível para identificar mudanças após o tratamento, sendo valioso no processo de avaliação, assim como na comparação dos tipos de tratamento ${ }^{10}$. Levando em conta a importância da especificidade do instrumento utilizado, a Sociedade Internacional de Continência recomendou a inclusão de um questionário de QV como complemento de medidas clínicas, sendo o King's Health Questionnaire (KHQ) um deles ${ }^{8,9}$, já traduzido e validado no Brasil ${ }^{10}$.

Há tempos sabe-se que o tratamento fisioterapêutico por meio do treinamento dos músculos do assoalho pélvico (TMAP), eletroestimulação vaginal e reforço com cones vaginais apresenta resultados expressivos na melhora dos sintomas de IU em até $85 \%$ dos casos 5 . A necessidade de avaliação mais ampla de como e quanto determinada doença/disfunção ou intervenção afeta a QV é fundamental em serviços de saúde. Diante do exposto, o objetivo deste estudo foi analisar a influência do tratamento fisioterapêutico na $\mathrm{QV}$ em mulheres com IU e a sua eficácia para a contenção da perda urinária.

\section{METODOLOGIA}

Neste estudo quase experimental foram incluídas $55 \mathrm{mu}-$ lheres com diagnóstico médico (clínico) de IU. As que se encaixaram nos critérios de seleção foram encaminhadas consecutivamente para atendimento fisioterapêutico 
por médicos do ambulatório de Uroginecologia do Hospital São Lucas (HSL) da Pontifícia Universidade Católica do Rio Grande do Sul (PUCRS), de setembro de 2006 a maio de 2010.

Os critérios de exclusão foram: participação em tratamento fisioterapêutico adicional ou qualquer tipo de atividade física estruturada e planejada durante o período do estudo, em adição àquelas previstas no protocolo de pesquisa; doenças mais incapacitantes que a IU (pneumopatias e cardiopatias severas, doenças neurológicas e/ou oncológicas) ou cirurgia para correção da IU prévia. Após inclusão no estudo, nenhuma mulher foi excluída.

As avaliações e a intervenção foram realizadas junto ao Serviço de Fisioterapia do HSL. As avaliações foram realizadas pelo mesmo avaliador, em raras ocasiões isso não aconteceu também com as sessões de tratamento. Os dados foram coletados após aprovação do Comitê de Ética e Pesquisa da PUCRS (Registro 06/03194) e assinatura do termo de consentimento livre e esclarecido.

O estudo se deu em três fases: avaliação inicial (anamnese e medições); intervenção; e avaliação final, sendo que na última eram repetidas as medições iniciais (avaliação da função da musculatura do assoalho pélvico - FMAP e aplicação do KHQ) assim que as participantes relatavam estar continentes, satisfeitas com o tratamento e/ou expressavam o desejo de pará-lo, o que, em alguns casos, aconteceu antes do total máximo de 15 sessões previsto no protocolo de pesquisa.

A avaliação da FMAP foi realizada por meio da manobra bidigital com a participante em decúbito dorsal com membros inferiores fletidos. Com luva lubrificada, o examinador introduziu o segundo e terceiro dedos por 3 a $4 \mathrm{~cm}$ dentro do canal vaginal e solicitou à paciente que contraísse e mantivesse a contração dos músculos ao redor de seus dedos. A FMAP foi avaliada com base na escala de Ortiz ${ }^{11}$, que a gradua de 0 (sem função perineal objetiva, nem mesmo à palpação) a 5 (função perineal objetiva e com resistência opositora por mais de cinco segundos à palpação).

A QV foi avaliada pelo KHQ autopreenchido, o qual é composto por 21 questões divididas em 8 domínios: percepção geral de saúde, impacto da IU, limitações de atividades diárias, limitações físicas, limitações sociais, relacionamento pessoal, emoções e sono/disposição. Existem ainda duas outras escalas independentes neste estudo utilizamos apenas a que avalia a presença e a intensidade dos sintomas urinários. O KHQé pontuado por cada um de seus domínios, cujos escores variam de 0 a 100, levando em conta que quanto maior a pontuação, pior a $\mathrm{QV}^{10}$.

A intervenção consistiu de uma única sessão semanal, por um máximo de 15 semanas. Os atendimentos foram divididos em duas partes, eletroestimulação (dez minutos) e TMAP. A eletroestimulação foi realizada com aparelho Dualpex 961 Uro (QUARK, Piracicaba, São Paulo - Brasil) e os parâmetros da corrente variaram de acordo com o tipo de IU apresentado: Heterodinia $2 \mathrm{~K} / 10 \mathrm{~Hz}$ para IU de urgência (IUU); Kots $2 \mathrm{~K} / 50$ $\mathrm{Hz}$, tempo de sustentação de 6 segundos, tempo de repouso de 12 segundos para IU de esforço (IUE). Para a IU mista (IUM), 10 e $50 \mathrm{~Hz}$ foram utilizados intercaladamente, isto é, numa semana era aplicada corrente de $10 \mathrm{~Hz}$ (IUU) e na outra de $50 \mathrm{~Hz}$ (IUE). A intensidade foi ajustada conforme a tolerância das pacientes, até o máximo de $60 \mathrm{~mA}$.

O TMAP se deu por meio de exercícios de abdução (faixa elástica) e adução (bola) na posição sentada e em decúbito dorsal, com contração da musculatura do assoalho pélvico (MAP). Em seguida, era feito o exercício de ponte, com ativação da MAP. Todos os exercícios envolveram contrações isotônicas e isométricas (mantidas por seis segundos), com uma série de dez repetições para cada tipo de exercício.

O número total de sessões e o protocolo de atendimento foram baseados na casuística do Serviço de Fisioterapia do HSL, onde o estudo foi desenvolvido, corroborados pelos achados de Lorenzo Gómez et al. ${ }^{12}$, os quais relataram melhora de $80 \%$ de suas pacientes após 12 semanas de tratamento.

\section{Tamanho amostral e análise estatística}

O cálculo do tamanho amostral (45 mulheres) foi realizado por meio do Programas para Epidemiologistas (PEPI) com base na resposta observada em estudo prévio $^{13}$, com significância de 95\% e erro máximo de $20 \%$.

Os dados foram analisados com o uso do software SPSS 17.0 e o nível de significância adotado (a) foi de 5\%. A simetria dos dados foi investigada pelo teste de Kolmogorov-Smirnov e as proporções de uma mesma variável comparadas pelo teste do $\chi^{2}$. Além disso, antes e após a intervenção, as variáveis contínuas foram comparadas pelo teste de Wilcoxom. 


\section{RESULTADOS}

A amostra foi composta por 55 mulheres com média de

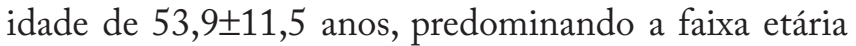
entre 51 e 60 anos. A maioria tinha baixa escolaridade, era casada e residia fora de Porto Alegre (Tabela 1).

A maior parte delas realizou exclusivamente partos normais $(63,5 \%)$, foi submetida à episiotomia $(65,9 \%)$ e apresentou prolapso (70,9\%). Em média, elas fizeram $2,6 \pm 1,6$ partos, sendo que 11 mulheres registraram os dois tipos, vaginal e cesárea. Do total de 122 partos, 101 foram vaginais e 21 cesáreas.

Apresentaram prolapso 39 mulheres: 30 de grau 1; sete de grau 2; e dois de grau 3. Do total, apenas duas não relataram melhora com o tratamento.

Em relação às demais características clínicas (Tabela 2), $36,4 \%$ das mulheres apresentaram IUE, 12,7\% IUU e 50,9\% IUM, sendo essa última significativamente maior $(\mathrm{p}<0,001)$.

As mulheres sofreram de IU durante 7 anos (mediana), antes de buscar tratamento, sendo que $75 \%$ delas apresentaram IU entre 4 e 10 anos. A maioria perdia urina por meio de jatos e gotas, principalmente devido a tosse ou espirro (Tabela 2).

Foram necessárias, em média, 13 sessões de tratamento para que $91 \%$ das mulheres se declarassem continentes ou satisfeitas com o tratamento (Tabela 2). Dez delas realizaram entre 5 e 10 sessões e todas relataram estar continentes após o tratamento. Outras 10 pacientes fizeram entre $11 \mathrm{e}$ 13 sessões; 11 fizeram 14; e as 24 restantes completaram as 15 sessões previstas no protocolo de pesquisa.

Após a intervenção, foi detectada melhora significativa $(\mathrm{p}<0,001)$ da FMAP (Tabela 2).

Apenas 3 mulheres não relataram melhora da perda urinária após a intervenção. Casadas, de baixa

Tabela 1. Características sociodemográficas da amostra

\begin{tabular}{|c|c|}
\hline \multirow{2}{*}{ Variáveis } & Total $(n=55)$ \\
\hline & $\mathrm{n}(\%)$ \\
\hline \multicolumn{2}{|l|}{ Idade (anos) } \\
\hline Média土desvio padrão & $53,9 \pm 11,5$ \\
\hline Mínimo-máximo & $35-87$ \\
\hline \multicolumn{2}{|l|}{ Grau de instrução } \\
\hline Não alfabetizada & $6(10,9)$ \\
\hline Ensino fundamental incompleto/completo & $32(58,2)$ \\
\hline Ensino médio incompleto ou superior & $17(30,9)$ \\
\hline \multicolumn{2}{|l|}{ Estado civil } \\
\hline Casada & $35(63,6)$ \\
\hline Solteira/separada/viúva & $20(36,4)$ \\
\hline \multicolumn{2}{|l|}{ Procedência } \\
\hline Porto Alegre & $21(38,2)$ \\
\hline Fora de Porto Alegre & $34(61,8)$ \\
\hline
\end{tabular}

escolaridade, residiam fora de Porto Alegre ( $\cong 140 \mathrm{~km}$ ) e fizeram apenas parto normal. Duas tinham prolapso (Grau 1 e 2). Uma delas fez 13 sessões de tratamento (IU por 21 anos; 68 anos), outra fez 14 (IU por 18 anos; 51 anos) e a outra 15 (IU por 5 anos; 42 anos). Para a mais jovem, o impacto da IU permaneceu o mesmo, enquanto as outras duas relataram diminuição maior que 50\%. A mais velha apresentou melhora na percepção geral da saúde, enquanto as outras duas não notaram diferença. Ela e a segunda mais velha relataram diminuição da gravidade da perda urinária e apresentaram aumento da FMAP. Para a mais jovem aumentaram a gravidade da perda urinária, suas limitações físicas e o impacto negativo da IU em suas relações pessoais e sociais, enquanto a FMAP não se alterou.

Em quase todos os domínios do KHQ (Tabela 3) foi detectada melhora significativa: impacto da

Tabela 2. Características clínicas da amostra

\begin{tabular}{|c|c|}
\hline \multirow{2}{*}{ Variáveis } & Total $(n=55)$ \\
\hline & $\mathrm{n}(\%)$ \\
\hline \multicolumn{2}{|l|}{ Diagnóstico médico } \\
\hline Incontinência urinária de esforço & $20(36,4)$ \\
\hline Incontinência urinária de urgência & $7(12,7)$ \\
\hline Incontinência urinária mista & $28(50,9)$ \\
\hline \multicolumn{2}{|l|}{ Tempo de incontinência (anos) } \\
\hline Mediana $\left(Q_{1}-Q_{3}\right)$ & $7,0(4,0-10,0)$ \\
\hline Mínimo-máximo & $1-31$ \\
\hline \multicolumn{2}{|l|}{ Quantidade de urina perdida } \\
\hline Gotas & $15(27,3)$ \\
\hline Jatos & $28(50,9)$ \\
\hline Miccção completa & $12(21,8)$ \\
\hline \multicolumn{2}{|l|}{ Situações de perda de urina } \\
\hline Tosse & $47(85,5)$ \\
\hline Espirro & $47(85,5)$ \\
\hline Risada & $30(54,5)$ \\
\hline Salto & $30(54,5)$ \\
\hline Orgasmo & $16(29,1)$ \\
\hline Caminhada & $30(54,5)$ \\
\hline Bexiga cheia (não segura) & $3(5,4)$ \\
\hline Levantamento de peso/ força & $7(12,7)$ \\
\hline Durante atividade física & $2(3,6)$ \\
\hline Outros & $4(5,4)$ \\
\hline \multicolumn{2}{|l|}{ Número de sessões de tratamento } \\
\hline Média土DP & $13,04 \pm 2,7$ \\
\hline Mínimo-máximo & $5-15$ \\
\hline \multicolumn{2}{|l|}{ Situação final } \\
\hline Continente/satisfeita & $50(90,9)$ \\
\hline Melhorou & $2(3,6)$ \\
\hline Não melhorou & $3(5,4)$ \\
\hline \multicolumn{2}{|l|}{ Teste bidigital - Mediana (Q1-Q3) } \\
\hline Antes do tratamento & $4(3-4)$ \\
\hline Depois do tratamento & $5(4-5)$ \\
\hline
\end{tabular}

Q1-Q3: intervalo interquartil; DP: desvio padrão 
Tabela 3. Escores dos domínios do King's Health Questionnaire, antes e depois do tratamento fisioterapêutico para incontinência urinária

\begin{tabular}{|c|c|c|c|}
\hline \multirow[t]{2}{*}{ Domínios } & \multicolumn{2}{|c|}{$\begin{array}{c}\text { Avaliações } \\
\text { Mediana (Q1-Q3) }\end{array}$} & \multirow[t]{2}{*}{ Valor $\mathrm{p}^{*}$} \\
\hline & Antes & Depois & \\
\hline Percepção geral de saúde & $50,0(25,0-50,0)$ & $25,0(25,0-50,0)$ & 0,234 \\
\hline Impacto da incontinência & $66,7(33,3-100,0)$ & $33,3(33,3-66,7)$ & 0,002 \\
\hline $\begin{array}{l}\text { Limitações das atividades } \\
\text { diárias }\end{array}$ & $33,3(33,3-66,7)$ & $33,3(0,0-50,0)$ & $<0,001$ \\
\hline Limitações físicas & $50,0(16,7-66,7)$ & $16,7(0,0-50,0)$ & $<0,001$ \\
\hline Limitações sociais & $33,3(0,0-55,6)$ & $11,1(0,0-22,2)$ & $<0,001$ \\
\hline Relações pessoais & $33,3(0,0-45,8)$ & $0,0(0,0-33,3)$ & 0,005 \\
\hline Emoções & $44,4(22,2-77,8)$ & $22,2(0,0-33,3)$ & $<0,001$ \\
\hline Sono e disposição & $33,3(16,7-50,0)$ & $33,3(16,7-33,3)$ & 0,001 \\
\hline Medidas de gravidade & $66,7(46,7-73,0)$ & $46,7(26,7-73,3)$ & 0,001 \\
\hline
\end{tabular}

*Teste de Wilcoxon; $\mathrm{Q}_{1}-\mathrm{Q}_{\text {: }}$ intervalo interquart

incontinência, limitações das atividades diárias, limitações físicas, limitações sociais, relações pessoais, emoções, sono e disposição e medidas de gravidade. Somente a percepção geral da saúde não apresentou diferença estatística significativa.

\section{DISCUSSÃO}

Após a intervenção, com exceção da percepção de saúde, houve melhora significativa em todos os domínios da QV, inclusive no impacto da IU nas limitações das atividades diárias e nas físicas das participantes, achados corroborados por outros estudos ${ }^{10,14}$. Eles provavelmente refletem o fato de que, assim que passam a ter melhor controle urinário, as mulheres se sentem menos preocupadas com eventuais episódios de incontinência e menos restritas na realização das atividades cotidianas, ocupacionais e físicas, especialmente aquelas com estilo de vida mais ativo ${ }^{10,14}$.

Todavia, não foi observada melhora estatística significativa na percepção geral da saúde, confirmando achados de Capelini et al. ${ }^{15}$ e sugerindo que, provavelmente, essas mulheres não consideram a IU uma doença ${ }^{3}$, haja visto a demora em buscar tratamento.

Predominaram mulheres entre 51 e 60 anos, fase da vida na qual a sintomatologia se exacerba ${ }^{4}$, o que provavelmente fez com que elas buscassem tratamento após anos de IU, corroborando o tempo de acometimento de 7 anos.

Neste estudo foram adotados métodos conservadores de avaliação e tratamento, de baixo custo, sem os efeitos colaterais das medicações e sem as intercorrências decorrentes das cirurgias ${ }^{9}$, em que a maioria relatou melhora e/ou cessação da perda urinária, confirmando o sucesso da intervenção e corroborando achados de outros estudos ${ }^{7,16}$. Estas informações são particularmente relevantes quando se considera que na rede pública de saúde é incomum o acesso ao tratamento fisioterapêutico para a IU. Se em uma capital esse acesso é restrito, ele é muito mais nas cidades do interior, como pôde ser visto nesta amostra, na qual quase $62 \%$ das participantes viajavam para fazer um atendimento curto ( $\cong 25$ minutos), que poderia ser facilmente oferecido na rede pública onde residiam caso fossem contratados fisioterapeutas.

Outro fator relevante no planejamento de ações em saúde que visem mulheres com IU é a baixa escolaridade das que dependem da saúde pública, conforme verificado neste e em outro estudo com usuárias da rede pública ${ }^{17}$. Deve-se ter em mente que há possíveis dificuldades de entendimento entre usuárias com menor escolaridade.

A presença de prolapso não poderia ter impedido a melhora das 3 mulheres que não evoluíram com o tratamento, visto que 1 não apresentava prolapso e, dentre aquelas com prolapso (39/55), 2 não relataram melhora. Estes achados corroboram outros que afirmam que o prolapso ser de diferentes graus não afeta a eficácia do tratamento fisioterapêutico da $\mathrm{IU}^{18,19}$. É possível que para essas mulheres fosse necessário um tratamento mais intenso e/ou prolongado, impossível em uma pesquisa. Não se pode descartar, contudo, a possibilidade de influência nos resultados por outras características além das investigadas.

A despeito dos achados relevantes e em consonância com a literatura ${ }^{1,27,12,13,15,16}$, o presente estudo apresenta limitações: ausência de grupo controle e amostragem sequencial não homogênea de mulheres com três tipos de IU e de diferentes faixas etárias. Porém, teríamos dificuldade em completar um estudo controlado, pois tanto a casuística do departamento no qual foi desenvolvido, quanto o estudo piloto realizado nos alertaram para possível dificuldade em recrutar o tamanho amostral necessário (45 mulheres/grupo; total de 90). Esta foi uma decisão acertada, posto que, em quase 4 anos de coleta, apenas 55 mulheres se encaixaram nos critérios de seleção. 
Não obstante, atingimos o nosso objetivo utilizando métodos de mensuração e de tratamento simples, práticos e de baixo custo, o que significa que o protocolo proposto aqui pode ser facilmente implementado na rede pública de saúde.

\section{CONCLUSÃO}

Os resultados deste estudo permitem concluir que o tratamento fisioterapêutico é eficaz não só para a melhora ou cessação da perda urinária, como também para a melhora da QV.

\section{REFERÊNCIAS}

1. Zanetti MR, Castro R de A, Rotta AL, Santos PD, Sartori M, Girão MJ. Impact of supervised physiotherapeutic pelvic floor exercises for treating female stress urinary incontinence. Sao Paulo Med J. 2007:125(5):265-9.

2. Beuttenmüller L, Cader SA, Macena RHM, Araujo NS, Nunes EFC, Dantas EHM. Contração muscular do assoalho pélvico de mulheres com incontinência urinária de esforço submetidas a exercícios e eletroterapia: um estudo randomizado. Fisioter Pesqui. 2011;18(3):210-6.

3. Silva L, Lopes MHBM. Incontinência urinária em mulheres: razões da não procura por tratamento. Rev Esc Enferm USP. 2009;43(1):72-8.

4. Lopes MHBM, Higa R. Restrições causadas pela incontinência urinária à vida da mulher. Rev Esc Enferm USP. 2006:40(1):34-41.

5. NICE Clinical Guidelines. Urinary incontinence: the management of urinary incontinence in women. RCOG Press. 2006;40:1-14.

6. Pedro AF, Ribeiro J, Soler ZASG, Bugdan AP. Qualidade de vida de mulheres com incontinência urinária. SMAD. 2011;7(2):63-70.

7. Honório GJS, Parucker NBB, Virtuoso JF, Krüger AP, Tonon SC, Ferreira R. Análise da qualidade de vida em mulheres com incontinência urinária antes e após tratamento fisioterapêutico. ACM Arq Catarin Med. 2009;38(4):43-9.
8. Dedicação AC, Haddad M, Saldanha MES, Driusso P. Comparação da qualidade de vida nos diferentes tipos de incontinência urinária feminina. Rev Bras Fisioter. 2009;13(2):116-22.

9. Tamanini JTN, D’Ancona CAL, Botega NJ, Rodrigues Netto Jr N. Validação do "King's Health Questionnaire" para o português em mulheres com incontinência urinária. Rev Saúde Pública. 2003;37(2):203-11.

10. Fonseca ESM, Camargo ALM, Castro RA, Sartori MGF, Fonseca MCM, Lima GR, et al. Validação do questionário de qualidade de vida (King's Health Questionnaire) em mulheres brasileiras com incontinência urinária. Rev Bras Ginecol Obstet. 2005;27(5):235-42.

11. Ortiz OC, Nunez FC. Valoración dinâmica de la disfunción perineal de clasificación. Boletim Sociedad Latino Americana Uroginecologia y Cirurgia Vaginal. 1994;:17-9.

12. Lorenzo Gómez MF, Silva Abuín JM, García Criado FJ, Geanini Yagüez A, Urrutia Avisrror M. Tratamiento de la incontinencia urinaria de esfuerzo con Biofeedback perineal con electrodos de superficie. Actas Urol Esp. 2008;32(6):629-36.

13. Goode PS, Burgio KL, Locher JL, Roth DL, Umlauf MG, Richter HE, et al. Effect of behavioral training with or without pelvic floor electrical stimulation on stress incontinence in women: a randomized controlled trial. JAMA. 2003;290(3):345-52.

14. Kelleher CJ, Cardozo LD, Khullar V, Salvatore S. A new questionnaire to assess the quality of life of urinary incontinent women. $\mathrm{Br} J$ Obstet Gynaecol. 1997;104(12):1374-9.

15. Capelini MV, Riccetto CL, Dambros M, Tamanini JT, Herrmann V, Muller V. Pelvic floor exercises with biofeedback for stress urinary incontinence. Int Braz J Urol. 2006;32(4):462-9.

16. Carneiro EF, Araujo NS, Beuttenmüll L, Vieira PC, Cader SA, Rett M, et al. Las características anatomofuncionales del suelo pélvico y la calidad de vida de mujeres con incontinencia urinaria de esfuerzo sometidas a ejercicios perineales. Actas Urol Esp. 2010;34(9):788-93.

17. Figueiredo EM, Lara JO, Cruz MC, Quintão DMG, Monteiro MVC. Perfil sociodemográfico e clínico de usuárias de serviço de Fisioterapia Uroginecológica da rede pública. Rev Bras Fisioter. 2008;12(2):136-42.

18. Knorst MR, Cavazzotto K, Henrique M, Resende TL. Physical therapy intervention in women with urinary incontinence associated with pelvic organ prolapse. Rev Bras Fisioter. 2012;16(2):102-7.

19. Ghroubi S, Kharrat O, Chaari M, Ayed BB, Guermazi M, Elleuch $\mathrm{MH}$. Apport du traitement conservateur dans la prise en charge du prolapsus urogénital de bas grade. Le devenir après deux ans. Physique. 2008:51(2):96-102. 